\title{
PRIMORDIAL CIRCUMSTELLAR DISKS IN BINARY SYSTEMS: EVIDENCE FOR REDUCED LIFETIMES
}

\author{
Lucas A. Cieza ${ }^{1,7}$, Deborah L. Padgett ${ }^{2}$, Lori E. Allen ${ }^{3}$, Caer E. McCabe ${ }^{2}$, Timothy Y. Brooke ${ }^{2}$, Sean J. Carey ${ }^{2}$, \\ Nicholas L. Chapman ${ }^{4}$, Misato Fukagawa ${ }^{5}$, Tracy L. Huard ${ }^{6}$, Alberto Noriga-Crespo ${ }^{2}$, Dawn E. Peterson ${ }^{3}$, and \\ LUISA M. REBULL ${ }^{2}$ \\ ${ }^{1}$ Institute for Astronomy, University of Hawaii at Manoa, Honolulu, HI 96822, USA; lcieza@ifa.hawaii.edu \\ ${ }^{2}$ Spitzer Science Center, MC 220-6, California Institute of Technology, Pasadena, CA 91125, USA \\ ${ }^{3}$ Harvard-Smithsonian Center for Astrophysics, 60 Garden Street, MS 42, Cambridge, MA 02138, USA \\ 4 Jet Propulsion Laboratory, California Institute of Technology, 4800 Oak Grove Drive, MS 301-429, Pasadena, CA 91109, USA \\ 5 Osaka University, 1-1 Machikaneyama, Toyonaka, Osaka 560-0043, Japan \\ ${ }^{6}$ Department of Astronomy, University of Maryland, College Park, MD 20742, USA \\ Received 2009 March 3; accepted 2009 March 19; published 2009 April 16
}

\begin{abstract}
We combine the results from several multiplicity surveys of pre-main-sequence stars located in four nearby starforming regions with Spitzer data from three different Legacy Projects. This allows us to construct a sample of 349 targets, including 125 binaries, which we use to to investigate the effect of companions on the evolution of circumstellar disks. We find that the distribution of projected separations of systems with Spitzer excesses is significantly different ( $P \sim 2.4 \mathrm{e}-5$, according to the $\mathrm{K}-\mathrm{S}$ test for binaries with separations less than $400 \mathrm{AU}$ ) from that of systems lacking evidence for a disk. As expected, systems with projected separations less than 40 AU are half as likely to retain at least one disk than are systems with projected separations in the 40-400 AU range. These results represent the first statistically significant evidence for a correlation between binary separation and the presence of an inner disk ( $r \sim 1 \mathrm{AU})$. Several factors (e.g., the incompleteness of the census of close binaries, the use of unresolved disk indicators, and projection effects) have previously masked this correlation in smaller samples. We discuss the implications of our findings for circumstellar disk lifetimes and the formation of planets in multiple systems.
\end{abstract}

Key words: binaries: general - circumstellar matter - infrared: stars - planetary systems: protoplanetary disks stars: pre-main sequence

Online-only material: machine-readable table

\section{INTRODUCTION}

Early multiplicity surveys of pre-main-sequence (PMS) stars in nearby star-forming regions have established that most lowmass stars in the solar neighborhood form in multiple systems (e.g., Leinert et al. 1993; Simon et al. 1995). Understanding the effect of multiplicity on the evolution of primordial circumstellar disks, the birthplace of planets, is therefore crucial to understand the potential for planet formation in most of the stars in the Galaxy. Recent models suggest that, starting from a disk of planetary embryos and planetesimals, planets can form and survive around individual members of binary systems with separations as small as $5 \mathrm{AU}$ (Quintana et al. 2007) as well as in orbits around both members of very close $(r<0.3 \mathrm{AU})$ binary systems (Quintana \& Lissauer 2006). However, it is still unclear how the presence of a close companion affects the evolution of the accretion disk, and whether or not the early disruption of the primordial disk is the limiting factor for the formation of planets in multiple systems.

Recent Spitzer studies (Padgett et al. 2006a; Cieza et al. 2007) found that up to $50 \%$ of the youngest weak-line T Tauri stars (age $\sim 1 \mathrm{Myr}$ ) show photospheric emission in the mid-IR, which implies that their planet-forming regions are extremely depleted of dust at this early age. One possible explanation for a very early disk dissipation is the efficient formation of planets in a less than $1 \mathrm{Myr}$ timescale (e.g., Boss 2000). Another possibility is the disruption of the disk due to the presence of close companions. The outer disks around the individual stars in a binary system are expected to be tidally truncated at a fraction

\footnotetext{
Spitzer Fellow.
}

( 0.3-0.5) of the binary separation (Papaloizou \& Pringle 1977) and it has been suggested that the truncation of the disk limits the amount of material that can be accreted and effectively shortens the accretion timescale in close binaries (Ghez et al. 1993).

Observational studies searching for a connection between close binaries and accelerated disk dissipation have so far yielded mixed and inconclusive results. Ghez et al. (1993) performed a speckle imaging survey of 69 stars in Taurus and Ophiuchus and concluded that the incidence of close binaries $(r<50 \mathrm{AU})$ in nonaccreting PMS stars (i.e., weakline T Tauri stars, WTTSs) is enhanced with respect to that of accreting objects (i.e., classical T Tauri stars, CTTSs). However, subsequent studies have failed to confirm this conclusion. Leinert et al. (1993) and Kohler \& Leinert (1998) performed larger speckle surveys in Taurus and concluded that both the total binary fraction and the distribution of projected binary separations of CTTSs and WTTSs are indistinguishable from each other. More recently, Ratzka et al. (2005) performed a speckle multiplicity survey of 158 PMS stars in Ophiuchus, the largest to date, and found a trend in the sense that objects with spectral energy distributions (SEDs) suggesting the presence of a disk have fewer companions but at smaller projected separations than that of objects with SEDs consistent with bare stellar photospheres.

Even though the truncation of disks in binary systems has very solid theoretical grounds, it has been repeatedly argued that, as long as disks in multiple systems can be replenished by an external reservoir (e.g., a circumbinary disk and/or an envelope), binaries will constrain the sizes of disks, but not 
necessarily reduce accretion disk lifetimes (Bouvier et al. 1997; Prato \& Simon 1997; Ghez et al. 1997; Mathieu et al. 2000). As a result, the lack of a statistically significant difference in the multiplicity and/or the binary separation of stars with and without disk indicators have lead many authors to conclude that multiplicity does not affect the lifetimes of circumstellar disks and/or planet formation (e.g., Simon \& Prato 1995; Armitage et al. 2003; Monin et al. 2007; Pascucci et al. 2008). Here we study a sample of 349 PMS stars, including 125 binaries with Spitzer data, and show that companions, at the peak of their separation distribution $(\sim 30 \mathrm{AU})$, do in fact shorten the lifetimes of circumstellar disks.

\section{THE SAMPLE}

We collected projected binary separations from near-IR multiplicity surveys of PMS stars located in the following nearby $(d<160 \mathrm{pc})$ star-forming regions: Ophiuchus (Simon et al. 1995; Prato 2007; Ratzka et al. 2005), Taurus (Leinert et al. 1993; Simon et al. 1995; Kohler \& Leinert 1998), Chameleon I (Lafrenière et al. 2008), and Corona Australis (Köhler et al. 2008).

Most of the surveys were performed using speckle imaging (Leinert et al. 1993; Kohler \& Leinert 1998; Ratzka et al. 2005; Köhler et al. 2008), but we also include results from lunar occultation (Simon et al. 1995), radial velocity (Prato 2007), and adaptive optics (Lafrenière et al. 2008) surveys. The speckle and adaptive optics surveys are sensitive to binaries down to projected separations similar to the $K$-band diffraction limits of the 3.5-8.2 m telescope used ( $\sim 0.06-0$ ' 13$)$, while the lunar occultation observations can detect binaries with separations as small as $0^{\prime \prime} 005$. The radial velocity surveys are of course sensitive to binaries at arbitrarily small separations, but are much less sensitive to wider binaries. As a result, our sample is highly heterogeneous in terms of completeness. However, as it will discussed in Section 4, the detection biases of the different surveys included in our study are unlikely to affect our conclusions.

Using their Two Micron All Sky Survey coordinates and a $2^{\prime \prime}$ matching radius, we searched for the Spitzer fluxes of all the targets from the surveys discussed above in the catalogs produced by the Cores to Disks (Evans et al. 2003), Taurus (Padgett et al. 2006b), and Gould Belt (Allen et al. 2007) Legacy Projects. We focus on the IRAC 3.6 and $8.0 \mu \mathrm{m}$ fluxes because (1) these Spitzer Legacy surveys are sensitive enough to reach the stellar photospheres of virtually all of the multiplicity targets in all IRAC bands, but not so at the MIPS wavelengths, and (2) the [3.6]-[8.0] color is the best disk indicator of all IRAC colors. We found 3.6 and $8.0 \mu \mathrm{m}$ fluxes with $\mathrm{S} / \mathrm{N}>5$ for 349 of the multiplicity targets, including 125 binaries. Their coordinates, projected separations (in the case of binaries), and Spitzer fluxes are listed in Table 1.

\section{RESULTS}

\subsection{Disk Identification}

In order to investigate the effect of binaries in the lifetime of circumstellar disks, we first need to establish the presence or absence of a circumstellar disk in each one of the systems in our sample. We do so by using the Spitzer colors as a disk indicator, as shown in Figure 1. There is a clear break in the color distribution of the sample around [3.6]-[8.0] $=$ 0.8. Thus, we consider systems with [3.6]-[8.0] $<0.8$ to be

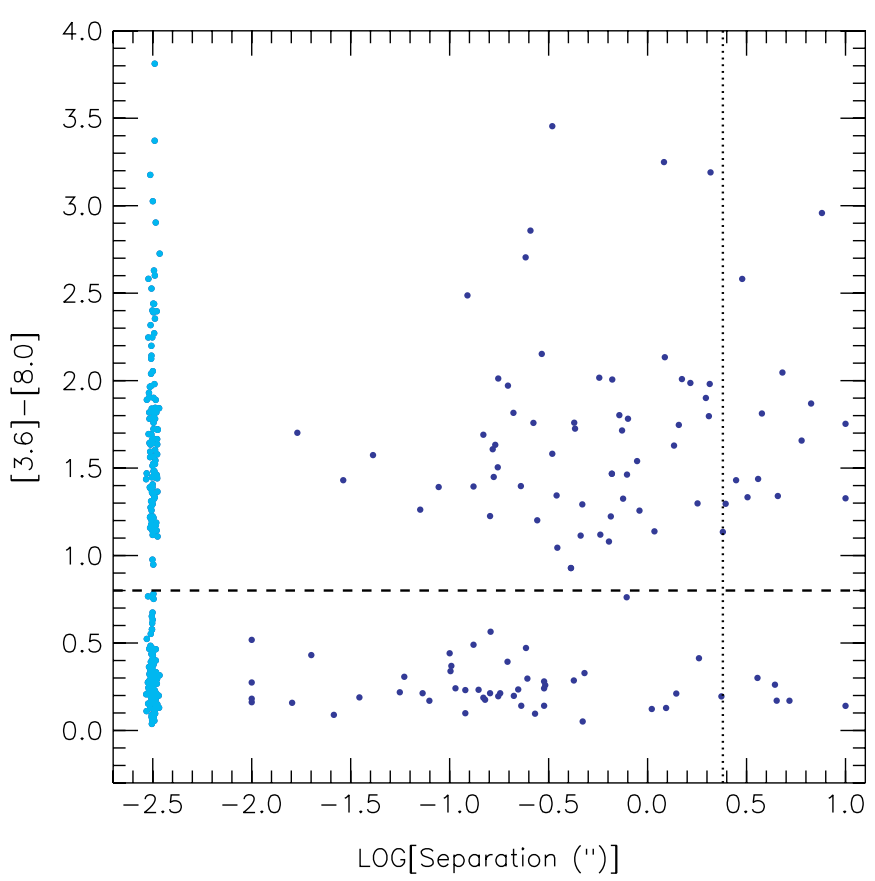

Figure 1. [3.6]-[8.0] vs. projected separation for our entire sample to illustrate our disk identification criterion. Systems with [3.6]-[8.0] $<0.8$ are considered to be diskless, while systems with [3.6]-[8.0] $>0.8$ are considered to harbor $a t$ least one disk. The dotted vertical line corresponds to 2 ." 4 , the angle sustained by 2 IRAC pixels. Only the few objects to the right of this line are likely to be resolved by Spitzer. Spectroscopic binaries have been assigned a separation of 0.01 . Single stars, shown in light blue, have been assigned a logarithmic separation of -2.5 plus very small random offsets to better show the density of objects at a given color.

diskless and systems with [3.6]-[8.0] $>0.8$ to harbor at least one circumstellar disk.

Given the distances involved (125-160 pc) and Spitzer's limited resolutions (2".0 FWHM at $8.0 \mu \mathrm{m})$, the vast majority of the multiple systems remain unresolved. As a result, except for very wide separation systems, Spitzer provides no information on whether the IR excess originates from one or both of the components in a binary system. The dotted vertical line in Figure 1 corresponds to 2 ". 4 , the size of 2 IRAC pixels, which is the radius of the photometry apertures for the Taurus Legacy Project data we use. The lower $\mathrm{S} / \mathrm{N}$ components of multiple objects detected within 2 pixels of each other have been dropped from their catalogs. The Cores to Disks and Gould Belt teams performed point-spread function fitting photometry, but objects less than 2 pixels apart are still unlikely to be resolved.

From Figure 1, we find that 186 of the 349 objects listed in Table 1 have an IR excess indicating the presence of a disk, of which 72 are known to be binaries and 114 are apparently single stars. Combining the multiplicity and disk identification information, we find that the disk fraction of multiple stars is marginally larger than that of stars that appear to be single $(57.6 \pm 4 \%$ versus $50.9 \pm 3 \%)$. Taken at face value, this result seems to imply that multiplicity has no effect on the evolution of circumstellar disks. However, as it will be shown in the following sections, this initial result can easily be understood in terms of the incompleteness and biases of the multiplicity surveys and the limitations of the disk-identification method.

\subsection{The Separation Distributions of Stars with and without a Disk}

The theoretical expectation of the effect of multiplicity on circumstellar disks is that, by tidally truncating each others 
Table 1

Multiplicity and Spitzer Data

\begin{tabular}{|c|c|c|c|c|c|c|c|c|c|c|c|c|c|}
\hline Name & $\begin{array}{c}\text { R.A. } \\
\text { (J2000) }\end{array}$ & $\begin{array}{c}\text { Decl. } \\
(\mathrm{J} 2000)\end{array}$ & Reg. & $\begin{array}{c}\text { Sep } \\
\left({ }^{\prime \prime}\right)\end{array}$ & $\operatorname{Ref}^{\mathrm{a}}$ & $\begin{array}{c}F_{3.6} \\
(\mathrm{mJy})\end{array}$ & $\begin{array}{c}\text { Error }_{3.6} \\
(\mathrm{mJy})\end{array}$ & $\begin{array}{c}F_{4.5} \\
(\mathrm{mJy})\end{array}$ & $\begin{array}{c}\text { Error }_{4.5} \\
(\mathrm{mJy})\end{array}$ & $\begin{array}{c}F_{5.8} \\
(\mathrm{mJy})\end{array}$ & $\begin{array}{c}\text { Error }_{5.8} \\
(\mathrm{mJy})\end{array}$ & $\begin{array}{c}F_{8.0} \\
(\mathrm{mJy})\end{array}$ & $\begin{array}{c}\text { Error }_{8.0} \\
(\mathrm{mJy})\end{array}$ \\
\hline V1095 Tau & 63.3090 & 28.3196 & Tau & & 1 & $08 e+02$ & $3.95 \mathrm{e}-01$ & $7.17 \mathrm{e}+01$ & $2.36 \mathrm{e}-01$ & $4.96 \mathrm{e}+01$ & & $2.73 e+01$ & $9.75 e-02$ \\
\hline V1096 Tau & 63.3635 & 28.2736 & Tau & $\cdots$ & 1 & $59 e+02$ & $6.58 \mathrm{e}-01$ & $2.28 \mathrm{e}+02$ & $.54 \mathrm{e}-01$ & $1.56 \mathrm{e}+02$ & 5.87 & $.44 \mathrm{e}+01$ & e-01 \\
\hline V773 Tau & 63.5538 & 28.2034 & Tau & 0.170 & 1 & $10 e+03$ & $1.75 \mathrm{e}+00$ & $1.03 \mathrm{e}+03$ & $2.02 \mathrm{e}+00$ & $1.02 \mathrm{e}+03$ & $1.42 \mathrm{e}+00$ & $1.13 e+03$ & $e+00$ \\
\hline FM Tau & 63.5566 & 28.2137 & Tau & $\ldots$ & 1 & $63 e+02$ & $3.71 \mathrm{c}$ & $1.53 e+02$ & 3.74 & $1.31 \mathrm{e}+02$ & 6.10 & $1.73 e+02$ & e-01 \\
\hline FN Tau & 63.5608 & 28.4661 & Tau & $\cdots$ & 1 & $2.59 e+02$ & & $2.43 e+02$ & $5.47 \mathrm{e}-01$ & & & $3.22 \mathrm{e}+02$ & $6.67 \mathrm{e}-01$ \\
\hline CW Tau & 63.5708 & 28.1827 & Tau & & 1 & & & & $2.55 \mathrm{e}+00$ & & & & $1.15 \mathrm{e}+00$ \\
\hline CX Tau & 63.6994 & 26.8031 & Tau & $\cdots$ & 1 & & & & & & & $1.42 \mathrm{e}+02$ & $3.63 e-01$ \\
\hline V1098 Tau & 63.6999 & 27.8763 & Tau & 0.470 & 1 & & & & $4.42 \mathrm{e}-01$ & & & $8.21 \mathrm{e}+01$ & $1.70 \mathrm{e}-01$ \\
\hline FO Tau & 63.7053 & 28.2085 & Tau & 0.165 & 1 & & & & & & & & $6.70 \mathrm{e}-01$ \\
\hline V1068 Tau & 64.1171 & 28.1266 & Tau & $\cdots$ & 1 & $1.50 \mathrm{e}+02$ & $3.95 \mathrm{e}-01$ & $9.66 \mathrm{e}+01$ & $2.20 \mathrm{e}-01$ & $6.99 \mathrm{e}+01$ & $2.40 \mathrm{e}-01$ & $3.86 \mathrm{e}+01$ & $1.21 \mathrm{e}-01$ \\
\hline
\end{tabular}

Notes.

a References: (1) Leinert et al. (1993); (2) Simon et al. (1995); (3) Lafrenière et al. (2008); (4) Prato (2007); (5) Ratzka et al. (2005); (6) Köhler et al. (2008).

(This table is available in its entirety in a machine-readable form in the online journal. A portion is shown here for guidance regarding its form and content.)

outer disks, close companions limit the amount of circumstellar material that can be accreted and hence reduce the lifetimes of their disks (Papaloizou \& Pringle 1977). Since the dispersal timescale of a truncated disk is given by the viscous timescale at the truncation radius, one expects the lifetimes of disks in binary systems to be a strong function of the binary physical separation. This prediction can be tested by investigating the disk fraction as a function of binary projected separation, or conversely, the distributions of binary separation of systems with and without a disk. Such distributions are shown in Figure 2, where the measured separations have been converted into projected physical separations (in AU) using the following distances: 125 for Ophichus (Loinard et al. 2008), $130 \mathrm{pc}$ for Corona Australis (Casey et al. 1998), $140 \mathrm{pc}$ for Taurus (Torres et al. 2007), and $160 \mathrm{pc}$ for Chameleon I (Whittet et al. 1997).

Figure 2 clearly shows that targets without an excess tend to have companions at smaller separations than targets with an excess indicating the presence of a disk. The disk fraction of the systems with separations less than $40 \mathrm{AU}$ is $38.2 \% \pm 6 \%$, while the disk fraction of systems with separations in the 40 $400 \mathrm{AU}$ range is $77.8 \pm 7 \%$. This difference in the disk fractions is $4.3 \sigma$. This is a robust result as a two-sided KolmogorovSmirnov $(\mathrm{K}-\mathrm{S})$ test shows that there is only a $2.4 \mathrm{e}-5$ probability that the distributions of binary separations of targets with and without disks have been drawn from the same parent population. Targets with projected separations greater than $400 \mathrm{AU}$ have been excluded from these calculations because they are likely to be resolved by Spitzer and therefore require a different statistical analysis than the rest of the sample.

For binary systems with separations smaller than the Spitzer beam, the disk fractions estimated above are not an accurate representation of the true disk fractions of the individual components of the systems. Assuming that each component of a binary system has the same individual probability, $\mathrm{DF}_{\text {ind }}$, of retaining a disk, the resulting fraction of systems with an IR excess, $\mathrm{DF}_{\mathrm{sys}}$, is given by the following equation: $\mathrm{DF}_{\mathrm{sys}}=1-(1-$ $\left.\mathrm{DF}_{\text {ind }}\right)^{2}$. Based on this formula, the disk fraction of the individual components of binary systems with projected separations less than $40 \mathrm{AU}$ is $21.4 \pm 5 \%$, while that of systems with separations in the $40-400 \mathrm{AU}$ range is $52.9 \pm 7 \%$, a disk fraction that is undistinguishable from that of apparently single stars, $50.9 \pm$ $3 \%$. In reality, $\mathrm{DF}_{\text {ind }}$ is unlikely to be exactly the same for both components of a binary system, especially if their mass ratio is

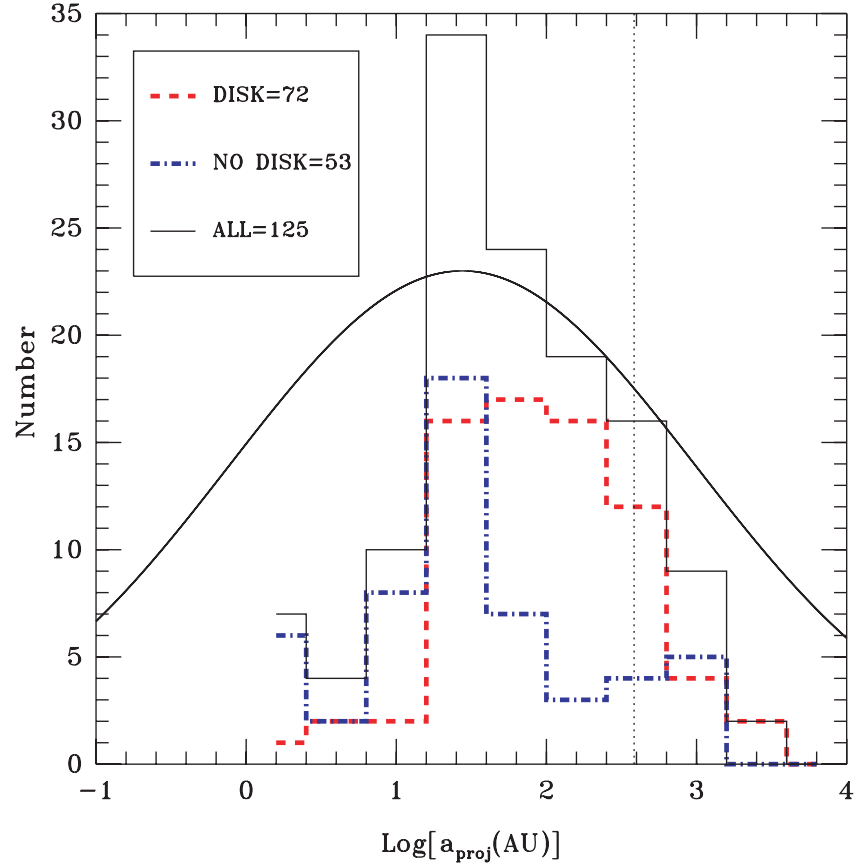

Figure 2. Histogram of projected separations for targets with and without Spitzer excesses indicating the presence of a disk. Systems without an excess clearly tend to have smaller separations. The solid curve represents the distribution of binaries in solar-type stars (Duquennoy \& Mayor, 1991). The census of companions is still highly incomplete for separations $\lesssim 20$ AU. The vertical dotted line at $X=2.58=\operatorname{LOG}(384 \mathrm{AU})$ corresponds to the resolution of 2 IRAC pixels (2".4) at $160 \mathrm{pc}$, the distance of the farthest regions in our sample. Systems to the right of this line are likely to be resolved by Spizter. This explains their lower disk fraction, as we are measuring their $\mathrm{DF}_{\text {ind }}$ instead of $\mathrm{DF}_{\text {sys }}$.

high, but the above calculation illustrates well the limitations of the disk fractions derived from unresolved disk indicators.

\section{DISCUSSION AND CONCLUSIONS}

Although we interpret the strong correlation between disk fraction and binary separation as an evidence for reduced disk lifetimes in close binary systems, the census of binaries in our sample is incomplete, especially at small separations (see Figure 2). Therefore, such a correlation could also arise if the older star-forming regions in our sample were observed with the techniques most sensitive to tight companions. In that case, 
Table 2

Properties of the Sample

\begin{tabular}{|c|c|c|c|c|c|c|c|c|c|c|c|}
\hline \multirow[t]{2}{*}{ Sample } & \multicolumn{4}{|c|}{$\begin{array}{c}\text { No. of Targets by } \\
\text { Technique }\end{array}$} & \multirow{2}{*}{$\begin{array}{c}\text { Targets } \\
\text { (Total No.) }\end{array}$} & \multirow{2}{*}{$\begin{array}{l}\text { Sin } \\
\text { (No.) }\end{array}$} & \multirow{2}{*}{$\begin{array}{l}\text { Bin } \\
\text { (No.) }\end{array}$} & \multirow{2}{*}{$\begin{array}{l}\text { Disk } \\
\text { (No.) } \\
\end{array}$} & \multirow{2}{*}{$\begin{array}{c}\text { Diskless } \\
\text { (No.) }\end{array}$} & \multirow{2}{*}{$\begin{array}{l}\text { DF } \\
(\%) \\
\end{array}$} & \multirow[t]{2}{*}{ Relative Age } \\
\hline & SP & $\mathrm{AO}$ & LO & RV & & & & & & & \\
\hline Tau Region & 65 & 0 & 46 & 0 & 84 & 43 & 41 & 54 & 30 & $64.3 \pm 5$ & youngest \\
\hline Oph Region & 141 & 0 & 33 & 19 & 158 & 108 & 50 & 83 & 75 & $52.5 \pm 4$ & young \\
\hline Cham I Region & 0 & 91 & 0 & 0 & 91 & 65 & 26 & 45 & 46 & $49.5 \pm 5$ & old \\
\hline CrA Region & 16 & 0 & 0 & 1 & 16 & 8 & 8 & 4 & 12 & $25.0 \pm 11$ & oldest \\
\hline LO+RV sample & 0 & 0 & 79 & 20 & 98 & 50 & 48 & 57 & 41 & $58.2 \pm 5$ & \\
\hline $\mathrm{SP}+\mathrm{AO}$ sample & 222 & 91 & 0 & 0 & 313 & 202 & 111 & 172 & 141 & $55.0 \pm 3$ & \\
\hline
\end{tabular}

Notes. $\mathrm{SP}+\mathrm{AO}+\mathrm{LO}+\mathrm{RV}$ is sometimes greater than the total number of targets because some overlap exists among some of the subsamples.

the close binaries in our sample would be systematically older than the wide binaries and thus would have lower disk fractions. We investigate this possibility by examining the relative ages of the stars in the four different regions included in our study. Instead of adopting ages from the literature, which are known to be model dependent, we derive their relative ages from their disk fractions. Table 2 shows that the targets from Cham I and Ophuichus have very similar disk fractions, but that the disk fraction of Taurus objects is clearly larger than that of CrA targets. Table 2 also shows that (1) virtually all the lunar occultation and radial velocity data, which are the most sensitive to close companions, come from the two youngest regions, and (2) the disk fraction of the lunar occultation plus radial velocity samples $(\mathrm{LO}+\mathrm{RV})$ are almost identical to that of the speckle plus adaptive optics samples ( $\mathrm{SP}+\mathrm{AO})$. These two facts strongly suggest that our results are not affected by the incompleteness and detection biases of our heterogeneous sample. We, therefore, conclude that the correlation between disk fraction and binary separation is due to the effect close binaries have on primordial disk lifetimes.

\subsection{Implications for Disk Lifetimes}

It has already been shown that $\lesssim 50-100 \mathrm{AU}$ separation binaries tend to have less (sub)millimeter emission than single stars or wider binaries (Osterloh \& Beckwith 1995; Jensen et al. 1996; Andrews \& Williams 2005). This implies lower disk masses for close binaries, but does not rule out the existence of small $(r<30 \mathrm{AU})$ disks with surface densities large enough to allow the formation of planets (Mathieu et al. 2000). Since Spitzer IRAC data probe circumstellar distances of the order of $1 \mathrm{AU}$, our results show that close binaries not only reduce the sizes of disks, but also their lifetimes.

The fraction of stars with disks as a function of age observed in nearby star-forming regions shows that there is a very wide range of primordial disk lifetimes. Some stars lose their disks well within the first Myr, while others retain their primordial disks for up to $10 \mathrm{Myr}$ (Haisch et al. 2001; Cieza et al. 2007). The results from the previous section strongly suggest that reduced disk lifetimes in binary systems can account for a significant part of the observed overall dispersion in disk lifetimes.

The distribution of physical separations, $a$, in solar-type PMS binaries is expected to peak around $30 \mathrm{AU}$ (Duquennoy \& Mayor 1991). However, the disks around most binary systems will have a truncation radius, $R_{T}$ given by $R_{T}=0.3-0.5 \times a \sim 10$ 15 AU (Papaloizou \& Pringle 1977). These truncation radii are $\sim 10$ times smaller than the typical radii of disks around single stars (Andrews \& Williams 2007). The viscous timescales for a disk with a power-law surface density profile of index $p$, is given by $t(r) \propto r^{2-p}$. Adopting $p=1$, which is consistent with both a steady state accretion disk and current observational constraints (Andrews \& Williams 2007) leads to $t(r) \propto r$. This implies that the lifetimes of disks around the individual components of most binary systems should be $\sim 10 \%$ of those of single stars. Assuming disk lifetimes of 3-5 Myr for single stars, this corresponds to disk lifetimes of $0.3-0.5 \mathrm{Myr}$ for binary systems at the peak of their separation distribution. These short disk lifetimes are broadly consistent with the disk fraction of $\sim 20 \%$ that we estimate for the individual components of binary systems with projected separations less than $40 \mathrm{AU}$ (see Section 3.2)

The rotation period distributions of PMS stars provide additional evidence for very early disk dissipation in a significant fraction of them. Rebull et al. (2004) and Cieza \& Baliber (2007) both found that the bimodal period distribution of the Orion Nebula Cluster can only be reproduced, in the context of the disk regulation of angular momentum paradigm, if disk lifetimes are themselves bimodal, with $30 \%-40 \%$ of the stars losing their regulating disks within less than $1 \mathrm{Myr}$ of their formation. Such bimodal distribution is in fact expected from a population combining single and binary stars.

\subsection{Implications for Planet Formation in Multiple Systems}

Since most stars in the Galaxy are likely to form in multiple systems, our results on the effect of multiplicity on circumstellar disk lifetimes have direct implications for planet formation. It is now almost universally accepted that terrestrial planets form through the continuous growth of solid particles. However, there is much less of a consensus on the formation mechanism of giant planets, with core accretion (Pollack et al. 1996) and gravitation instability (Boss 2000) being the two competing leading theories.

Even though recent core accretion models (e.g., Alibert et al. 2004) can reproduce the formation of a solid core massive enough $\left(\sim 10 M_{\oplus}\right)$ to start accreting a gaseous envelope within a few Myr, disk lifetimes of 0.3-0.5 Myr are most likely to represent a significant challenge to giant planet formation through core accretion in binary systems. Reduced accretion lifetimes impose a weaker but still significant obstacle to terrestrial planet formation. Gas drag is crucial for the growth of planetesimals into planetary embryos as it reduces the relative velocity of planetesimals to a regime in which accretion can take place (Xie \& Zhou 2008). In the absence of gas, planetesimals subjected to the gravitational perturbations of a binary system are expected to sustain relative velocities well in excess of the threshold at which erosion dominates over accretion (Marzari \& Scholl 2000). Thus, in order for terrestrial planets to efficiently 
form in binary systems, planetary embryos should be formed before the gas dissipates.

While it seems very likely that multiplicity disfavors terrestrial planet formation in general and giant planet formation through core accretion, its effect on giant planet formation through gravitational instability remains unclear. Depending on the details of the models, it has been argued that multiplicity both inhibits (e.g., Mayer et al. 2005) and enhances (e.g., Boss 2006) planet formation through gravitational instability.

Given the limited number of currently known exoplanets in binary systems, it is not yet possible to draw statistically significant results on the incidence of planets as a function of binary separation. However, there are already some indications that giant planets are in fact under-represented in binary systems with separations $\lesssim 50-100$ AU (Eggenberger et al. 2007; Bonavita \& Desidera 2007). If most giant planets form through core accretion, the findings presented herein would provide a natural explanation for such a result.

Even though many previous studies of smaller samples have searched for a connection between multiplicity and an accelerated disk dissipation (e.g., Leinert et al. 1993, Ghez et al. 1993; Simon \& Prato 1995; Ratzka et al. 2005; Bouwman et al. 2006; Monin et al. 2007), the results from this Letter represent the first statistically significant (i.e., over $3 \sigma$ ) evidence that binaries reduce the lifetimes of circumstellar disks. This is not too surprising considering that: (1) unresolved disk indicators only provide information on whether at least one of the components in a binary system has a disk, (2) the observed projected separations represent the minimum possible value of the true physical separations; thus many of the systems only appear to be tight binaries due to projection effects, and (3) the multiplicity surveys are highly incomplete for tight systems, where the effect on disk lifetimes is expected to be most severe. The combination of these three factors explains why a very large sample is necessary to reveal the effect of multiplicity on disk lifetimes.

We thank Jonathan Williams, Jonathan Swift, Michael Liu, and the anonymous referee for their valuable comments. Support for this work was provided by NASA through the Spitzer Fellowship Program under an award from Caltech. This work makes use of data obtained with the Spitzer Space Telescope, which is operated by JPL/Caltech, under a contract with NASA.

\section{REFERENCES}

Alibert, Y., Mordasini, C., \& Benz, W. 2004, A\&A, 417, L25

Allen, L., et al. 2007, BAAS, 38, 881

Andrews, S. M., \& Williams, J. P. 2005, ApJ, 631, 1134

Andrews, S. M., \& Williams, J. P. 2007, ApJ, 659, 705

Armitage, P. J., Clarke, C. J., \& Palla, F. 2003, MNRAS, 342, 1139

Bonavita, M., \& Desidera, S. 2007, A\&A, 468, 721

Boss, A. P. 2000, ApJ, 536, L101

Boss, A. P. 2006, ApJ, 641, 1148

Bouvier, J., Rigaut, F., \& Nadeau, D. 1997, A\&A, 323, 139

Bouwman, J., et al. 2006, ApJ, 653, L57

Casey, B. W., Mathieu, R. D., Vaz, L. P. R., Andersen, J., \& Suntzeff, N. B. 1998, AJ, 115, 1617

Cieza, L., \& Baliber, N. 2007, ApJ, 671, 605

Cieza, L., et al. 2007, ApJ, 667, 308

Duquennoy, A., \& Mayor, M. 1991, A\&A, 248, 485

Eggenberger, A., et al. 2007, A\&A, 474, 273

Evans, N. J., II, et al. 2003, PASP, 115, 965

Ghez, A. M., Neugebauer, G., \& Matthews, K. 1993, AJ, 106, 2005

Ghez, A. M., White, R. J., \& Simon, M. 1997, ApJ, 490, 353

Haisch, K. E., Jr., E. A., Lada, \& Lada, C. J. 2001, ApJ, 553, L153

Jensen, E. L. N., Mathieu, R. D., \& Fuller, G. A. 1996, ApJ, 458, 312

Kohler, R., \& Leinert, C. 1998, A\&A, 331, 977

Köhler, R., et al. 2008, A\&A, 488, 997

Lafrenière, D., et al. 2008, ApJ, 683, 844

Leinert, C., et al. 1993, A\&A, 278, 129

Loinard, L., Torres, R. M., Mioduszewski, A. J., \& Rodríguez, L. F. 2008, ApJ, 675, L29

Marzari, F., \& Scholl, H. 2000, ApJ, 543, 328

Mathieu, R. D., Ghez, A. M., Jensen, E. L. N.., \& Simon, M. 2000, in Protostars and Planets IV, ed. V. Mannings, A. P. Boss, \& S. S. Russell (Tucson, AZ: Univ. Arizona Press), 703

Mayer, L., Wadsley, J., Quinn, T., \& Stadel, J. 2005, MNRAS, 363, 641

Monin, J.-L., et al. 2007, in Protostars and Planets V, ed. V. B. Reipurth, D. Jewitt, \& K. Keil (Tucson, AZ: Univ. Arizona Press), 395

Osterloh, M., \& Beckwith, S. V. W. 1995, ApJ, 439, 288

Padgett, D. L., et al. 2006a, ApJ, 645, 1283

Padgett, D., et al. 2006b, BAAS, 38, 947

Papaloizou, J., \& Pringle, J. E. 1977, MNRAS, 181, 441

Pascucci, I., et al. 2008, ApJ, 673, 477

Pollack, J. B., Hubickyj, O., Bodenheimer, P., Lissauer, J. J., Podolak, M., \& Greenzweig, Y. 1996, Icarus, 124, 62

Prato, L. 2007, ApJ, 657, 338

Prato, L., \& Simon, M. 1997, ApJ, 474, 455

Quintana, E. V., \& Lissauer, J. J. 2006, Icarus, 185, 1

Quintana, E. V., et al. 2007, ApJ, 660, 807

Ratzka, T., Köhler, R., \& Leinert, C. 2005, A\&A, 437, 611

Rebull, L. M., Wolff, S. C., \& Strom, S. E. 2004, AJ, 127, 1029

Simon, M., \& Prato, L. 1995, ApJ, 450, 824

Simon, M., et al. 1995, ApJ, 443, 625

Torres, R. M., Loinard, L., Mioduszewski, A. J., \& Rodríguez, L. F. 2007, ApJ, 671, 1813

Whittet, D. C. B., et al. 1997, A\&A, 327, 1194

Xie, J.-W., \& Zhou, J.-L. 2008, ApJ, 686, 570 